Proyecciones

Vol. 27, $\mathrm{N}^{o}$ 1, pp. 29-61, May 2008.

Universidad Católica del Norte

Antofagasta - Chile

\title{
ON THE RETROSECTION THEOREM *
}

\author{
RUBÉN HIDALGO \\ UNIVERSIDAD TÉCNICA FEDERICO SANTA MARÍA, CHILE
}

Received: May 2006. Accepted: March 2008

\begin{abstract}
We survey some old and new results related to the retrosection theorem and some of its extensions to compact Klein surfaces, stable Riemann surfaces and stable Klein surfaces.
\end{abstract}

Keywords : Riemann Surfaces, Klein surfaces, Schottky Groups

Subjclass : 2000, 30F10, 30F40

\footnotetext{
*Partially supported by Project Fondecyt 1070271
} 


\section{Introduction}

We survey some old and new results related to the retrosection theorem and some of its extensions. Let $S$ be a closed Riemann surface of genus $g$. Classical retrosection theorem asserts that we may find a Schottky group $G$, with region of discontinuity $\Omega$, and a regular planar covering $P: \Omega \rightarrow S$ with $G$ as covering group; that is $G$ uniformizes $S$.

In order to extend classical retrosection theorem to the class of compact Klein surfaces, we need to find a natural extension of a Schottky group. An extended Schottky group is an extended Kleinian groups whose index two orientation preserving half is a Schottky group. By definition, an extended Schottky group contains orientation reversing transformations and the region of discontinuity of it is the same as for its index two Schottky subgroup. If $R$ is now a compact Klein surface, then the first extension of the retrosection theorem asserts the existence of an extended Schottky group $K$ with region of discontinuity $\Omega$ and a regular (branched at the borders) planar covering $Q: \Omega \rightarrow R$ with $K$ as covering group. We may see $R$ as the quotient $S /\langle\tau\rangle$ where $S$ is a closed Riemann surface and $\tau: S \rightarrow S$ is an anticonformal automorphism. If we denote by $G$ the index two orientation preserving half of $K$, then the above may be stated as the existence of a regular planar covering $P: \Omega \rightarrow S$ with $G$ as covering group so that $\tau$ lifts to an anticonformal automorphism of $\Omega$ under $P$. In the case that $R$ is closed, that is, when $\tau$ has no fixed points on $S$, the extended Schottky group has no reflections; its orientation reversing transformations are only glide-reflections and/or imaginary reflections. These extended Schottky groups are the Klein-Schottky groups.

The boundary of moduli space in the Deligne-Mumford's compactification corresponds to conformal classes of closed stable Riemann surfaces. In order to consider an extension of retrosection theorem to the class of stable Riemann surfaces, different from a closed Riemann surface, we need to consider some kind of deformations of Schottky groups. At this points, we deal with two types of degenerations. One degeneration provides geometrically finite Kleinian groups in the algebraic boundary of Schottky space; the noded Schottky groups. The other kind of degenerations is provided by first considering geometrically finite Kleinian groups in the algebraic border of a co-compact Fuchsian group and then by quotient them by suitable torsion free normal subgroup (in a similar way as to obtain Schottky groups as quotient of co-compact Fuchsian groups); the stable Schottky groups. Noded Schottky groups are a particular subclass of stable Schottky group. 
An extended region of discontinuity of an stable Schottky group is defined and the concept of a regular planar covering is given in order to see that every stable Riemann surface may be uniformized by a stable Schottky group. Moreover, they may be uniformized just only using noded Schottky groups. Some stable Schottky groups were already considered in [8].

Stable Klein surfaces are obtained as quotient of stable Riemann surfaces by some anticonformal involution. Uniformizations of stable Klein surfaces are given by certain degenerations (in some sense) of extended Schottky groups, called extended stable Schottky groups, and all stable Klein surfaces can be so uniformized, so this can be though as a retrosection theorem at the level of stable Klein surfaces.

This survey is structured as follows. In Section 2 we recall the definitions of (extended) Möbius transformations, Klein surfaces, (extended) Kleinian groups, geometrically finite Kleinian groups, (extended) function groups and the extended region of discontinuity. In Section 3 we recall Koebe's uniformization theorem of analytically finite Riemann surfaces by (extended) function groups. In Section 4 we recall the definition of a Schottky group and state the classical retrosection theorem, We also provided other equivalent definitions of Schottky groups and discuss some problems related to classical Schottky groups, including a conjecture due to Bers. In Section 5 we recall the definition of extended Schottky groups and, in particular, of a Klein-Schottky group. Then, we state the retrosection theorem for the class of compact Klein surfaces. In Section 6 we consider a retrosection theorem for closed Riemann surfaces with automorphisms, so that the automorphisms are reflected. In Section 7 we recall the definitions of stable Riemann surfaces, the Deligne-Mumford's compactification, quasiconformal deformation spaces, Teichmüller and Schottky spaces and real structures. In Section 8 we provide the definition of a stable Klein surface as the orbit space of a stable Riemann surface by an anticonformal involution. In Section 9 we define some degenerations of Schottky groups; noded Schottky groups and stable Schottky groups. Then, we state a retrosecton theorem for stable Riemann surfaces. In Section 10 we define some degenerations of extended Schottky groups; extended stable Schottky groups and extended noded Schottky groups. In Section 11 we provide a retrosection theorem for stable Klein surfaces. 


\section{Some preliminaries}

\subsection{Möbius and extended Möbius transformations}

Let us denote by $M$ the group of Möbius transformations and by $\widehat{M}$ the group generated by $M$ and $\tau(z)=\bar{z}$. The elements of $\widehat{M}-M$ are called extended Möbius transformations and correspond to the anticonformal automorphisms of $\widehat{C}$. By Poincaré extension, we may see $M$ (respectively, $\widehat{M}$ ) as the group of orientation preserving hyperbolic isometries (respectively, the total group of hyperbolic isometries) of of hyperbolic 3-space $\mathbf{H}^{3}$.

A loxodromic transformation is an orientation preserving isometry of $H^{3}$ that has exactly two fixed points, necessarily on the sphere at infinity; every loxodromic transformation is conjugate, in the group of isometries of $H^{3}$, to one of the form $z \rightarrow \lambda e^{i \theta} z$, where $\lambda$ is real and $\lambda>1$ (if moreover, $\theta \in 2 \pi \mathbf{Z}$, then we say that it is a hyperbolic transformation). A parabolic transformation is an orientation preserving isometry of $H^{3}$ that has exactly one fixed point, necessarily on the sphere at infinity; every parabolic transformation is conjugate, in the group of isometries of $H^{3}$, to $z \rightarrow z+1$. An elliptic transformation is an orientation preserving isometry with a hyperbolic line of fixed point; every elliptic transformation is conjugate to the transformation $z \rightarrow e^{i \theta} z$.

A glide reflection is an orientation reversing isometry of $H^{3}$ that has exactly two fixed points, necessarily on the sphere at infinity; every glide reflection is conjugate, in the group of isometries of $H^{3}$, to one of the form $z \rightarrow \lambda \bar{z}$, where $\lambda$ is real and $\lambda>1$. A pseudo-parabolic is an orientation reversing isometry of $H^{3}$ that has exactly one fixed point, necessarily on the sphere at infinity; every pseudo-parabolic is conjugate, in the group of isometries of $H^{3}$, to $z \rightarrow \bar{z}+1$. An imaginary reflection is an orientation reversing isometry of order two with exactly one fixed point, necessarily in the interior of hyperbolic space; every imaginary reflection is conjugate to the transformation $z \rightarrow-1 / \bar{z}$.

\subsection{Klein and Riemann surfaces}

A Klein surface is a surface on which the change of coordinates are either conformal or anticonformal (that is, a di-analytic structures) [2]. If all change of coordinates are conformal, then we are in the presence of a Riemann surface. Now on, we reserve the name Klein surface for one different from a Riemann surface, that is, there is at least some anticonformal local change of coordinates. In this way, Klein surfaces may be seen as quotients 
of Riemann surfaces by some anticonformal automorphisms of order two. A compact Klein surface is the quotient of a closed Riemann surface by some anticonformal involution. A Klein surface is said to have algebraic genus $g$ if it is the quotient of a closed Riemann surface of genus $g$ by an anticonformal involution.

\subsection{Kleinian and extended Kleinian groups}

A Kleinian group is a discrete subgroup of $\mathbf{M}$ and an extended Kleinian group is a subgroup $K$ of $\widehat{\mathbf{M}}$ so that its index two orientation preserving half $K^{+}$is a Kleinian group. If $K$ is a (extended) Kleinian group, then the Riemann sphere is divided into two disjoint sets: the region of discontinuity $\Omega(K)$ and the limit set $\Lambda(K)$.

We say that an extended Kleinian group $K$ acts freely or it is freely acting on its region of discontinuity if the stabilizer of any point of its region of discontinuity (in the Riemann sphere) is trivial. A freely acting extended Kleinian group may have non-trivial elements with fixed points in the interior of hyperbolic space (when we see it as a group of isometries of hyperbolic space), for instance, an imaginary reflection always has a fixed point on $\mathbf{H}^{3}$.

\subsection{Geometrically finite Klenian groups}

A geometrically finite Kleinian group is one for which there is a finite sided fundamental polyhedron when seen as a group of isometries of $\mathbf{H}^{3}$. Similarly, an extended Kleinian group $K$ is called geometrically finite if $K^{+}$is geometrically finite.

\subsection{Function groups}

A function group is given by a couple $(F, \Delta)$, where $F<\mathbf{M}$ is a finitely generated Kleinian group and $\Delta \subset \widehat{\mathbf{C}}$ is an invariant component of the region of discontinuity of $F$ [23]. In case $\Delta$ is a simply connected domain, we say that $(F, \Delta)$ is a $B$-group. An extended function group is a pair $(K, \Delta)$ for which $K$ is an extended Kleinian group and $\Delta$ is invariant under the action of $K$. An extended function group $(H, \Delta)$ for which $\Delta$ is simply connected is an extended B-group. At this point, let us note that if we consider a Fuchsian group $F$, acting on the unit disc so that its limit set is all of the unitary circle, and $\tau$ is the reflection on that unitary circle, then 
$K=\langle F, \tau\rangle$ is an extended Kleinian group with $K^{+}=F$ a function group, but $K$ is not an extended function group.

\subsection{Extended region of discontinuity}

Given a (extended) Kleinian group $G$ we define its extended region of discontinuity $\Omega^{e x t}(G)$ as the union of its region of discontinuity with its doublecusped parabolic fixed points [9] (see also section 9.1). The complement $\Lambda^{\text {ext }}(G)=\widehat{\mathbf{C}}-\Omega^{\text {ext }}(G)$ is called the extended limit set of $G$. Observe that if $G$ has no double-cusped parabolic fixed points (for instance, Schottky groups and co-compact Fuchsian groups), then $\Omega^{e x t}(G)=\Omega(G)$ and $\Lambda^{e x t}(G)-\Lambda(G)$.

We say that an (extended) Kleinian group $K$ is freely acting on its extended region of discontinuity if the stabilizer of any point of its extended region of discontinuity is trivial.

\section{Koebe's uniformization theorem}

Let $(K, \Delta)$ be a function group so that no non-trivial element of $K$ fixes a point in $\Delta$. Then, $R=\Delta / K$ is a Riemann surface and we have a natural regular planar holomorphic covering $P: \Delta \rightarrow R$.

An uniformization of a Riemann surface $S$ is a triple $(\Delta, F, P: \Delta \rightarrow S)$, where $(F, \Delta)$ is a function group and $P: \Delta \rightarrow S$ is a holomorphic regular covering with $F$ as cover group. As consequence of Ahlfors' finiteness theorem [1], we have that $S$ should be analytically finite, that is, is the complement of a finite set of points (possible empty) of a closed Riemann surface. Similarly, an uniformization of a Klein surface $S$ is a triple $(\Delta, F, P: \Delta \rightarrow S)$, where $(F, \Delta)$ is an extended function group and $P: \Delta \rightarrow S$ is a di-analytic regular covering with $F$ as cover group.

Theorem 1 (Koebe's uniformization theorem [18, 19]) Every analytically finite (Klein) Riemann surface can be uniformized by (extended) function groups.

Moreover, quasiconformal deformation theory asserts that if we have an (extended) function group $(F, \Delta)$ uniformizing a (Klein) Riemann surface $S$, then every (Klein) Riemann surface quasiconformally equivalent to $S$ can be also uniformized by a quasiconformal deformation of the previous (extended) function group. 


\section{Retrosection theorem for closed Riemann surfaces}

\subsection{Schottky groups}

A Schottky group of genus $g \geq 1$ is defined as the group generated by $g$ loxodromic transformations $A_{1}, \ldots, A_{g}$, with the following geometric restriction: should exist a collection of $2 g$ pairwise disjoint simple loops, say $C_{1}, C_{1}^{\prime}, \ldots, C_{g}$ and $C_{g}^{\prime}$, all of them bounding a common domain $D$ of connectivity $2 g$, so that $A_{j}\left(C_{j}\right)=C_{j}^{\prime}$ and $A_{j}(D) \cap D=\emptyset$, for all $j=1, . ., g$. The generators $A_{1}, \ldots, A_{g}$ are called a Schottky set of generators and the loops $C_{1}, C_{1}^{\prime}, \ldots, C_{g}, C_{g}^{\prime}$ a fundamental set of loops. A result due to V. Chuckrow [5] asserts that every set of free generators of a Schottky group is in fact a Schottky set of generators. A classical Schottky group is a Schottky group which admits a fundamental set of loops, for a suitable set of Schottky generators, being all of them circles of $\widehat{\mathbf{C}}$. Schottky groups were invented by Schottky (at least the classical ones), before Klein and Poincaré initiated the general theory on Kleinian groups. The name Schottky group was coined by Poincaré. The basic properties on Schottky groups were obtained after 1968 by $[5,6,22,25]$ just to mention a few of them.

\subsection{Retrosection theorem}

If we denote by $\Omega$ (respectively, by $\Lambda$ ) the region of discontinuity (respectively, the limit set) of a Schottky group $G$ of genus $g$, then $\Omega$ is connected and $\Lambda$ is empty for $g=0$, consists of two points for $g=1$ and it is a Cantor set for $g \geq 2$. Also, the quotient $\Omega / G$ turns out to be a closed Riemann surface of genus $g$. The reciprocal holds and it is known as (Koebe) retrosection theorem [18]. A modern proof of retrosection theorem is given in [3] with the help of quasiconformal deformation theory.

Theorem 2 (Retrosection theorem for closed Riemann surfaces) Every closed Riemann surface may be uniformized by a suitable Schottky group.

\subsection{Other equivalent definitions of a Schottky group}

A Schottky group $G$ of genus $g$ may also be defined as a geometrically finite Kleinian group which is purely loxodromic and is isomorphic to a free group of rank $g[5,23,25]$. Let us consider a regular planar covering $P: \Delta \rightarrow R$. We say that $P$ is a lowest regular planar covering of $R$ if each time we have another regular planar covering $Q: \Delta^{\prime} \rightarrow R$ and a continuous map $T: \Delta \rightarrow \Delta^{\prime}$ with $P=Q T$ (then $T$ is necessarily a regular planar 
covering map), then $Q$ is necessarily a homeomorphism. Schottky groups are known to be the covering groups providing the lowest (holomorphic) regular planar coverings of closed Riemann surfaces.

\subsection{Classical versus nonclassical Schottky groups}

L. Bers observed the existence of Schottky groups admitting Schottky generators for which there is no fundamental set of loops being all of them circles. It is then important to note that a classical Schottky group may have a Schottky set of generators for which there is no fundamental set of loops being circles. Examples of classical Schottky groups are those which are Fuchsian ones [4]. The theoretical existence of nonclassical Schottky groups is due to Marden [22]. This is done by comparison of the algebraic boundaries of the space of (marked) classical Schottky groups (classical Schottky space) with the space of (marked) Schottky spaces (Schottky space). The algebraic boundary of classical Schottky space consists of geometrically finite Kleinian groups and the boundary of Schottky space has geometrically infinity Kleinian groups. The first claim of a concrete example of a nonclassical Schottky group was dome by Zarrow [34] in 1975, but Sato [32] observed that such an example was classical in 1988. Later, in 1991, Yamamoto [33] constructed a correct example of a nonclassical Schottky group of genus 2. In [15] there is also a theoretical construction of infinitely many nonclassical Schottky groups for every genus.

\subsection{A conjecture due to Bers}

Let $S$ be a closed Riemann surface of genus $g \geq 2$. Retrosection theorem asserts the existence of Schottky groups that provide uniformizations of $S$. Unfortunately, there is no an explicit way to determine when two given Schottky groups (both of same genus) provide uniformizations of conformally equivalent Riemann surfaces. This problem makes hard a solution to the following classical problem due to Bers.

Conjecture 1. Every closed Riemann surface of genus $g \geq 2$ may be uniformized by a classical Schottky group.

It was known to Koebe [19] (see Theorem 5.5) that if the surface admits an anticonformal involution with fixed points, then it may be uniformized

by a classical Schottky group. This result is also an easy consequence of 
quasiconformal deformation theory. C. McMullen observed, by use of collar's lemma and modulus of ring domains, that if a closed Riemann surface of genus $g \geq 2$ admits a collection of $g$ pairwise disjoint, homologically independent, simple closed geodesics with sufficiently small hyperbolic lenghts, then it may be uniformized by a classical Schottky group.

\section{Retrosection theorem for compact Klein surfaces}

\subsection{Extended Schottky groups}

An extended Schottky group $G$ of genus $g$ is an extended Kleinian group whose index two orientation preserving half $G^{+}$is a Schottky group of genus $g$. If we denote by $\Omega$ the region of discontinuity of $G^{+}$, then we have that $\Omega$ is also the region of discontinuity of $G$. In this case, $S^{+}=$ $\Omega / G^{+}$is a closed Riemann surface of genus $g$ admitting an anticonformal automorphism $\tau: S^{+} \rightarrow S^{+}$of order 2 so that $S=S^{+} / \tau=\Omega / G$. In this way, $S$ is a compact Klein surface of algebraic genus $g$ with boundary if and only if $\tau$ acts with fixed points on $S^{+}$. We say that $S$ is uniformized by the extended Schottky group G. Some particular classes of extended Schottky groups are defined below.

\subsection{Real Schottky groups}

A real Schottky group is an extended Schottky group for which its index two orientation preserving Schottky group keeps invariant a circle on the Riemann sphere and which is generated by such a Schottky group and the reflection on the invariant circle.

\subsection{Klein-Schottky groups}

Let us consider an extended Schottky groups $G$, with region of discontinuity $\Omega$, so that $S=\Omega / G$ is a closed Klein surface, that is, it has no boundary. This asserts that $G$ cannot have reflections nor parabolics (then, neither can have pseudo-parabolics), in particular, the extended Schottky group $G$ acts freely on its region of discontinuity. These particular class of extended Schottky groups are called Klein-Schottky groups and can be constructed as follows [16]. Let $m$ and $n$ be non-negative integers, with $m+n>0$. Let $D$ be a region in the extended complex plane bounded by $m+2 n$ disjoint Euclidean circles. Label these circles as $B_{1}, \ldots, B_{m}, C_{1}, C_{1}^{\prime}, \ldots, C_{n}, C_{n}^{\prime}$. For $i=1, \ldots, m$, let $\sigma_{i}$ be an imaginary reflection mapping the inside of $B_{i}$ to its 
outside. For $j=1, \ldots, n$, let $\rho_{j}$ be a glide reflection mapping the inside of $C_{j}$ to the outside of $C_{j}^{\prime}$. It is easy to see that $G=\left\langle\sigma_{1}, \ldots, \sigma_{m}, \rho_{1}, \ldots, \rho_{n}\right\rangle$ is a combination theorem free product of the cyclic groups generated by these generators, and that $G^{+}$, the orientation preserving half of $G$ is a Schottky group (in fact, a classical Schottky group) of genus $g=(2 n+m-1)$; then, $G$ is a Klein-Schottky group of genus $g$. We call the group $G$, or any quasiconformal deformation of $G$, an $(m, n)$-Klein-Schottky group. It is immediate that any two $(m, n)$-Klein-Schottky groups define topologically equivalent uniformizations of topologically equivalent Klein surfaces.

\subsection{Retrosection theorem for compact Klein surfaces} 19])

Theorem 3 (Retrosection theorem for compact Klein surfaces [16, 17,

An extended Schottky group of genus $g$ uniformizes a Klein surface of algebraic genus $g$. Reciprocally, every Klein surface of algebraic genus $g$ can be uniformized by an extended Schottky group of genus $g$. The unbordered case correspond to exactly the Klein-Schottky groups. Moreover, if $X$ is a closed Klein surface of algebraic genus $g$, then, for each pair $(m, n)$ of nonnegative integers so that $g=2 n+m-1$, there is a $(m, n)$-Klein-Schottky group uniformizing $X$.

\subsection{The bordered case}

That every bordered compact Klein surface can be uniformized by a real Schottky group was already known to Koebe.

Theorem 4 (Retrosection theorem for bordered compact Klein surfaces [19]) Every bordered compact Klein surface may be uniformized by a suitable real Schottky group.

\section{Retrosection theorem with automorphisms}

The previous retrosection theorems may be seen as particular cases of a more general one which we explain in this section.

Let us consider a closed Riemann surface $S$ and a group $H<A u t(S)$, where $A u t(S)$ denotes the group of conformal/anticonformal automorphisms of $S$; we denote by $A u t^{+}(S)$ its subgroup of conformal automorphisms. In this case, $O=S / H$ turns out to be a compact orbifold, maybe with 
non-empty border, with a di-analytic structure (a conformal structure if $\left.H<\mathrm{Aut}^{+}(S)\right)$. Classical retrosection theorem states that $S$ may be uniformized by a Schottky group. We may ask for the existence of a Schottky uniformization for which $H$ lifts, that is, to find a Schottky group $G$, say with region of discontinuity $\Omega$, and a regular holomorphic covering $P: \Omega \rightarrow S$ with $G$ as covering group so that there is a (extended) Kleinian group $K$ containing $G$ as a normal subgroup and so that $K / G$ represents $H$ (this means that $S / H=\Omega / K$ ). If this holds, then we say that $H$ is a group of Schottky type.

For instance, if $H=\langle\tau\rangle$ is the group generated by a symmetry on $S$, then the last section asserts that $H$ is of Schottky type.

A collection of pairwise disjoint simple loops on $S$, say $L_{1}, \ldots, L_{k} \subset S$, is called a Schottky system of loops of $H$ if

(1) each connected component of $S-\cup_{j=1}^{n} L_{j}$ is a genus zero bordered surface; and

(2) the collection of loops $\left\{L_{1}, \ldots, L_{k}\right\}$ is invariant under the action of the group $H$.

We have the following necessary and sufficient condition for a group of automorphisms to be of Schottky type.

Theorem 5 ([13]) A group $H<A u t(S)$ of automorphisms of a closed Riemann surface $S$ of genus $g \geq 2$ is of Schottky type if and only if there is a Schottky system of loops of $H$

The case $H=\{I\}$ is the classical retrosection theorem and the case $H$ generated by a symmetry is the retrosection theorem for compact Klein surfaces.

Theorem 6 may be seen as a retrosection theorem for compact orbifolds. Let us consider a compact orbifold $O$ with a di-analytic structure. This means the existence of a (extended) function group $F$, with invariant component $\Delta$ of its region of discontinuity, so that $\Delta / F=O$. The group $F$ is finitely generated as consequence of Ahlfors finiteness theorem (applied to the orientation preserving subgroup of $F$ ). It follows from Selberg's lemma that $F$ has a finite index normal subgroup $F_{0}$. Its orientation preserving half is then also finite index normal subgroup of $F$, so we may assume $F_{0}$ a function group (all its transformations are orientation preserving ones). In this way, $S=\Delta / F_{0}$ is a closed Riemann surface admitting the group $H=K / F_{0}<\operatorname{Aut}(S)$. If $H$ is of Schottky type, then we have the existence of a (extended) Kleinian group $K$, with region of discontinuity $\Omega$, so that 
$\Omega / K=O$ and $K$ contains a Schottky group as a normal subgroup of finite index. The only problem with this is that the finite index normal subgroup $F_{0}$ is non unique and it may happens that a choice for $F_{0}$ produces a group which is not of Schottky type and probably we may find another for which the corresponding group $H$ is of Schottky type. In order to overcome this problem, we need to observe that Theorem 6 permits to provide a geometrical classification of all those (extended) Kleinian groups containing a Schottky group as a normal subgroup of finite index. Of course, this is not an easy task, but if we restrict ourselves to low index, then it can be carry out. Once this is done, it provides the types of orbifolds $O$ for which a choice of $F_{0}$ produxes a Schottky type group of automorphisms.

\section{Deformation spaces}

Before to continue with more extensions of the retrosection theorem to degenerate surfaces, we need to recall some definitions and facts.

\subsection{Stable Riemann surfaces}

A stable Riemann surface is a (not necessarily compact) connected complex space of dimension one $R$, that is, defined locally by a (finite) set of analytic functions, so that the singular points of $R$ (those points at which $R$ fails to be a two-dimensional real manifold) have a system of neighborhoods homeomorphic to the set $\left\{(z, w) \in \mathbf{C}^{2} ; z \cdot w=0,|z|<1,|w|<1\right\}$. These points are called the nodes of $R$, and we denote the set of them by $N(R)$ (the nodal set of $R$ ). We say that $R$ is a stable Riemann surface of genus $g$ if the following hold:

(1) If $g \geq 2$, then

(1.1) Every component $R_{i}$ of $R-N(R)$ is a Riemann surface of genus $g_{i}$ with $n_{i}$ deleted points so that $2 g_{i}-2+n_{i}>0$, and

(1.2) If $R_{1}, \ldots, R_{k}$ are the components of $R-N(R)$ and the number of nodes of $R$ is $m$, then $g=\sum_{i=1}^{k} g_{i}+m+1-k$.

(2) If $g=1$, then either $R$ is a Riemann surface of genus one, or $N(R)$ consists of exactly one point, say $p$, so that $R-\{p\}$ is a Riemann surface conformally equivalent to the punctured plane $\mathbf{C}^{*}=\mathbf{C}-\{0\}$.

(3) If $g=0$, then $R \cong \widehat{\mathbf{C}}$. 
If $R$ is a stable Riemann surface of genus $g$ and $N(R)=\emptyset$, then $R$ is a Riemann surface of genus $g$. Two stable Riemann surfaces $S$ and $R$ are said to be topologically equivalent if there is a homeomorphism $f: S \rightarrow R$. If the restriction of such a homeomorphism to the complement of the nodes $f: S-N(S) \rightarrow R-N(R)$ is bi-holomorphic, we say that $R$ and $S$ are conformally equivalent.

\subsection{Deligne-Mumford's compactification}

The moduli space $M_{g}$ of genus $g$ is by definition the set of conformal classes of closed Riemann surfaces of genus $g$. This is a complex analytic space with singularities, in fact, a quasi-algebraic projective variety. A compactification $\widehat{M}_{g}$ of $M_{g}$, called the Deligne-Mumford's compactification, is obtained after adding the conformal class of stable Riemann surfaces of genus $g$ and it turns out that $\widehat{M}_{g}$ has the structure of an algebraic projective variety of complex dimension $3 g-3$ if $g \geq 2$ [30].

\subsection{Quasiconformal deformation spaces}

Let us consider a (extended) function group $(K, \Delta)$. Associated to it we have the unit ball $L_{1}^{\infty}(K, \Delta)$ is given by the measurable functions $\mu: \widehat{\mathbf{C}} \rightarrow$ C so that $\|\mu\|_{\infty}<1, \mu \equiv 0$ in $\Delta^{c}$ and

$$
\mu(k(z))= \begin{cases}\frac{k_{z}(z)}{\overline{k_{z}(z)}} \mu(z), & \text { if } k \in K^{+} \\ \frac{k_{\bar{z}}(z)}{\overline{k_{\bar{z}}(z)}} \bar{\mu}(z), & \text { if } k \in K-K^{+}\end{cases}
$$

A equivalence relation $\sim$ is defined on $L_{1}^{\infty}(K, \Delta)$ as follows. If $\mu \in$ $L_{1}^{\infty}(K, \Delta)$, then we have a unique quasiconformal homeomorphism $W^{\mu}$ : $\widehat{\mathbf{C}} \rightarrow \widehat{\mathbf{C}}$, with complex dilation $\mu$, that fixes 0,1 and $\infty$. We have that for each $k \in K^{+}$(respectively, $k \in K-K^{+}$) the element $k^{\mu}=W^{\mu} k W^{\mu-1}$ is a Möbius transformation (respectively, extended Möbius transformation) if $k$ is Möbius transformation (respectively, and extended Möbius transformation). If we set $K^{\mu}=W^{\mu} K W^{\mu-1}$, then we have an isomorphism of groups

$$
\chi_{\mu}: K \rightarrow K^{\mu}: k \mapsto k^{\mu} .
$$

We say that $\mu_{1}, \mu_{2} \in L_{1}^{\infty}(K, \Delta)$ are equivalent $\left(\mu_{1} \sim \mu_{2}\right)$ if $\chi_{\mu_{1}}=\chi_{\mu_{2}}$. The quotient space

$$
Q(K, \Delta)=L_{1}^{\infty}(K, \Delta) / \sim
$$


is called the quasiconformal deformation space of $(K, \Delta)$. As the group $K$ is finitely generated, by definition, we have that $Q(K, \Delta)$ is:

(i) a real analytic manifold of finite dimension, if $K \neq K^{+}$; and

(ii) a complex manifold of finite dimension, if $K=K^{+}$.

The topology in $Q(K, \Delta)$ turns out to be the algebraic convergence one. General details on quasiconformal deformation spaces of Kleinian groups can be found in the book of S. Nag [31]. In the case that $G$ is a Schottky group of genus $g$, with region of discontinuity $\Omega$, its quasiconformal deformation space $Q(G)=Q(G, \Omega)$ is called a Schottky space, and it turns out to be a connected complex manifold of dimension

$$
\operatorname{dim}_{\mathbf{C}} Q(G)= \begin{cases}3 g-3, & g \geq 2 \\ 1, & g=1 \\ 0, & g=0\end{cases}
$$

As a consequence of the retrosection theorem, there is a natural holomorphic map from the Schottky space $Q(K)$ onto the moduli space $M_{g}$ of closed Riemann surfaces of genus $g$. Similarly, given an extended Schottky group $\Gamma$ of genus $g$, its quasiconformal deformation space $Q(\Gamma)=Q(\Gamma, \Omega)$ is called an extended Schottky space, which is a connected real manifold of real dimension

$$
\operatorname{dim}_{\mathbf{R}} Q(\Gamma)= \begin{cases}3 g-3, & g \geq 2 \\ 1, & g=1 \\ 0, & g=0\end{cases}
$$

If we have extended Schottky groups $\Gamma$ and $\Gamma^{*}$ both algebraically isomorphic, then we have a real analytic isomorphism between their respective deformation spaces.

\subsection{Teichmüller and Schottky space}

The quasiconformal deformation space of a B-group, uniformizing a closed Riemann surface of genus $g$, gives us a model of the universal cover space of $M_{g}$, that is, the Teichmüller space $T_{g}$. The quasiconformal deformation of a Schottky group of genus $g$ (that is, the Schottky space) gives us the Schottky cover of $M_{g}$. The real points on moduli space can be seen as the projections of the real points on the Schottky space. To obtain, at the level of Schottky groups, the Deligne-Mumford's compatification $\widehat{M}_{g}$, we need to add certain boundary points (in the algebraic convergence topology) of the 
Schottky space. Such boundary points correspond to the noded Schottky groups [9] and the stable Schottky groups, which proceed to define in a next section.

\subsection{Real structures}

Let $\Gamma$ be some extended Schottky group of genus $g$ and set $G=\Gamma^{+}$; which is a Schottky group of genus $g$. We have a natural real analytic imbedding of the extended Schottky space $Q(\Gamma)$ into the Schottky space $Q(G)$ by restriction of Beltrami coefficients of $\Gamma$ to $G$. If we write $\Gamma=\langle G, \tau\rangle$, then the extended Möbius transformation $\tau \in \Gamma$ induces an antiholomorphic involution $\tau^{*}$ on the Schottky space $Q(G)$, defined by

$$
\tau^{*}([\mu(z)])=\left[\frac{\tau_{\bar{z}}(z)}{\overline{\tau_{\bar{z}}(z)}} \bar{\mu}(z)\right] .
$$

The locus of fixed points of $\tau^{*}$ is exactly the embedding of $Q(\Gamma)$. In this way, we may identified the real points of the Klein real structure $\tau^{*}$ on the corresponding Schottky space with the extended Schottky space associated to the extended Schottky group $\Gamma$.

We have that real points represented by Klein-Schottky groups correspond to closed Klein surfaces and those represented by extended Schottky groups which are not Klein-Schottky groups correspond to bordered compact Klein surfaces.

\section{Stable Klein surfaces}

\subsection{Anticonformal involutions on stable Riemann surfaces}

An anticonformal involution of a stable Riemann surface $S$ is by definition a homeomorphism $\sigma: S \rightarrow S$ of order two so that on the complement of the nodes it is an anticonformal map.

\subsection{Stable Klein surfaces}

A surface of the form $S / \sigma$, where $S$ is a stable Riemann surface of genus $g$ and $\sigma$ is an anticonformal involution on $S$, is called a stable Klein surface of algebraic genus $g$. The image on $X=S / \sigma$ of a node on $S$ can be either: (i) a node (that is, it has a neighborhood homeomorphic to a node of $S$ ); (ii) a half-node (it has a neighborhood homeomorphic to the set $\left\{(z, w) \in \mathbf{C}^{2} ; z \cdot w=0,|z|<1,|w|<1, \operatorname{Im}(z) \geq 0, \operatorname{Im}(w) \geq 0\right\} ;$ (iii) 
a phantom node (it has a neighborhood homeomorphic to the unit disc). The collection of the nodes, half-nodes and phantom nodes of $X=S / \sigma$ is called the nodal set and denoted also by $N(X)$. Let us observe that each component of the complement of the nodal set of an stable Riemann surface $S$ of genus $g \geq 2$ is of hyperbolic type, that is, it has the hyperbolic plane as universal cover. It follows that each component of the complement of the nodal set of a stable Klein surface is also covered (as orbifold) by the hyperbolic plane.

Example 1. As the only stable Riemann surface of genus zero is the Riemann sphere, we have that the stable Klein surfaces of algebraic genus zero are the (i) real projective plane $\mathbf{R P}_{2}$ and (ii) the closed disc. As the stable Riemann surfaces of genus one are either a torus or a torus with one node, we have that the stable Klein surfaces of algebraic genus one are (i) the closed annuli, (ii) $\mathbf{R P}_{2}$ with one component border, (iii) the Klein bottle $\mathbf{R} \mathbf{P}_{2} \# \mathbf{R} \mathbf{P}_{2}$, (iv) a closed disc with a phantom node, (v) $\mathbf{R} \mathbf{P}_{2}$ with a phantom node and (vi) the closed set formed by the common part of two tangent different circles.

Stable Klein surfaces of algebraic genus $g$ may be though as the boundary points of the real structures on moduli space $M_{g}$. To describe those points at the level of Schottky groups, we need to consider certain groups, called extended stable Schottky groups, defined as those groups containing as index two subgroup a stable Schottky group.

\section{Stable and Noded Schottky Groups}

In this section we introduce two class of uniformization groups of stable Riemann surfaces, each one given by certain degeneration of Schottky groups.

The first kind of degenerations are given as follows. Let us consider a B-group $(F, \Delta)$ so that $\Delta / F$ is a closed Riemann surface of genus $g$. If $N \triangleleft F$ is so that $G=F / N$ is free group, then we have that $G$ is a Schottky group with region of discontinuity $\Omega=\Delta / N$. The reciprocal holds as consequence of the uniformization theorem. We may now consider some geometrically finite degenerations of the B-group $(F, \Delta)$ to obtain the noded $\pi_{g}$-groups (see section 9.1 ). This degenerations of $(F, \Delta)$ produces some degenerations of the Schottky group $G$, obtaining the stable Schottky groups (see section 9.2). Unfortunately, in general a stable Schottky group do not act on the Riemann sphere, but on trees of open dense subsets 
of Riemann spheres. Examples of stable Schottky groups were already considered By Gerritzen and Herrlich in [8]. Their definitions were purely topological, the one obtained in here are from the point of view of Kleinian groups.

The second kind of degenerations can be done considering geometrically finite (algebraic) degenerations of Schottky groups, producing in this way the noded Schottky groups [9] (see section 9.3). We may see noded Schottky groups as a subclass of stable Schottky groups: they are those stable Schottky groups acting on a Riemann sphere.

\subsection{Noded $\pi_{g}$ group}

A Kleinian group $G$ which is geometrically finite and algebraically isomorphic to the fundamental group of a closed orientable surface of genus $g$ will be called a noded $\pi_{g}$ group. We have seen in [10] that the region of discontinuity of a noded $\pi_{g}$ group $G$ is necessarily non-empty (this is an easy consequence of Marden's isomorphism theorem). Let $\Omega^{\text {ext }}(G)$ be its extended region of discontinuity and $\Omega(G)$ its region of discontinuity. We consider the cuspidal topology on this extended region, that is, the topology generated by the usual open sets in $\Omega(G)$ together the sets of the form $D_{1} \cup D_{2} \cup\{p\}$, where $p$ is a double cusped parabolic fixed point and $D_{1}$ and $D_{2}$ are Euclidean discs contained in $\Omega(G)$ and tangent at $p$. It is clear that a noded $\pi_{0}$ group is the trivial group and a $\pi_{1}$ group is a rank two Abelian group. B. Maskit observed in [28] that for $g \geq 2$ a noded $\pi_{g}$ group having no parabolics is necessarily a quasifuchsian group uniformizing a couple of closed Riemann surfaces of genus $g$ and, in particular, $\Omega(G)=\Omega^{e x t}(G)$ consists of exactly two simply connected invariant components. In the presence of parabolics elements this is essentially the same situation and we have the following simultaneous uniformization theorem for stable surfaces.

Theorem 6 [[10]] Let $G$ be a noded $\pi_{g}$ group, where $g \geq 2$. Then $\Omega^{e x t}(G)$ consists of exactly two components, each one simply connected and invariant, and the quotient of each of them by $G$ is a stable Riemann surface of genus $g$, where the quotient map is analytic outside the parabolic fixed points. Reciprocally, if $S_{1}$ and $S_{2}$ are any two stable Riemann surfaces of same genus $g \geq 2$, then there is a noded $\pi_{g}$ group $G$ so that $\Omega^{e x t}(G) / G=S_{1} \cup S_{2}$.

Remark 1. Some examples of noded $\pi_{g}$ groups are given by regular $B$ groups ( finitely generated Kleinian groups $G$ with a simply connected in- 
variant component $\Delta$ so that $\operatorname{Area}(\Delta / G)=\operatorname{Area}((\Omega(G)-\Delta) / G)[20])$ isomorphic to the fundamental group of some closed Riemann surface. Ahlfor's finiteness theorem [1] asserts that $\Omega(G) / G$ is a finite union of analytically finite Riemann surfaces. That a regular B-group is geometrically finite is given, for instance, in [26, 27].

\subsection{Stable Schottky groups}

Now we proceed to give the first class of groups that give uniformizations of stable Riemann surfaces that can be seen as certain class of degenerations of a Schottky group. Some of these groups where already considered in [8].

Let us start with some noded $\pi_{g}$ group $\Gamma$. Let $\Delta$ be one of the two components of $\Omega^{e x t}(\Gamma)$. Then $S=\Delta / \Gamma$ is a stable Riemann surface of genus $g$. Let $K$ be a normal subgroup of $\Gamma$ so that $G=\Gamma / K$ is a free group of rank $g$, and set $Z=\Delta / K$ with the quotient topology. We have that $Z$

is a stable Riemann surface so that its nodes are given by the projections of the double-cusped parabolic fixed points of $K$ inside $\Delta$. The group $G$ acts as a group of homeomorphisms on $Z$ and as a group of conformal automorfisms on the complement of its nodes. Let $P: Z \rightarrow S$ be the natural quotient map induced by the action of $G$. We have that $P$ turns out to be holomorphic in $(\Delta \cap \Omega(\Gamma)) / K$. We say that $(Z, G, P: Z \rightarrow S)$ is a stable Schottky uniformization of $S$ and that $G$ is a stable Schottky group of genus $\mathrm{g}$. Theorem 9.1 asserts the following:

Corollary 1 (Retrosection theorem for stable Riemann surfaces). Every stable Riemann surface of genus $g \geq 2$ can be uniformized by a suitable stable Schottky group. Moreover, if the surface $S$ has no nodes, that is, when $S$ is a closed Riemann surface, then the stable Schottky group is a Schottky group.

The above result asserts that the boundary points of $M_{g}$ inside the Deligne-Mumford's compactificaton $\widehat{M}_{g}$ can be uniformized by stable Schottky groups.

\section{Remark 2.}

(i) A stable $\pi_{1}$ group $G$ containing parabolics is, up to conjugation, generated by the transformations $A(z)=z+1$ and $B(z)=z+t$, where $\operatorname{Im}(t)>0$. We have that $\Omega^{e x t}(G)=\Omega(G)=\mathbf{C}$, that is, we have no 
double-cuped parabolic points. In this case, $K$ is a rank one parabolic subgroup, which we may assume to be generated by the transformations $A$. We have that $Z=\mathbf{C} /\langle A\rangle=\mathbf{C}-\{0\}$ and $G / K \cong \mathbf{Z}$ is a cyclic group generated by a loxodromic transformation which has as fixed points 0 and $\infty$. In this way, an stable Schottky groups of genus 1 is necessarily a Schottky group.

(ii) Let us consider a regular $B$-group $(\Gamma, \Delta)$ so that $\Delta / \Gamma$ is a closed Riemann surface $S$ of genus two and so that $\left(\Omega^{e x t}(\Gamma)-\Delta\right) / \Gamma$ uniformizes the stable Riemann surface of genus two with one dividing node and one non-dividing node at each complement of the first. Let $K$ be the smallest normal subgroup of $\Gamma$ containing the three primitive parabolics that define these three nodes. If $Z=\left(\Omega^{e x t}(\Gamma)-\Delta\right) / K$, then $\Gamma / K$ defines a stable Schottky group uniformizing $\left(\Omega^{\text {ext }}(\Gamma)-\Delta\right) / \Gamma$.

\subsection{Noded Schottky groups}

Now we proceed to the second class of degenerations of Schottky groups. A noded Schottky group of genus $g$ is a geometrically finite Kleinian group isomorphic to a free group of rank $g$. If $G$ is a noded Schottky group of genus $g$, then we have that $\Omega(G) \neq \emptyset, \Omega^{e x t}(G)$ is connected and that $S=\Omega^{e x t}(G) / G$ is an stable Riemann surface of genus $g$ [9]. The triple $\left(\Omega^{\text {ext }}(G), G, P: \Omega^{\text {ext }}(G) \rightarrow S\right)$ is called a noded Schottky uniformization of $S$. In this case, geometrically finiteness asserts that every parabolic fixed point of a noded Schottky group is double-cusped, in particular, $\Omega^{e x t}(G)$ is the union of $\Omega(G)$ with all parabolic fixed points. Noded Schottky groups are obtained as algebraic degenerations of Schottky groups [24].

\section{Remark 3.}

(i) We have that the a noded Schottky group of genus one is isomorphic to the cyclic group $\langle z \mapsto z+1\rangle$. Recall that we have already observed that there are no stable Schottky group of genus one except Schottky groups.

(ii) In the case $g \geq 2$, noded Schottky groups are a particular class of stable Schottky groups as they correspond to the case where $K$ (the normal subgroup of the noded $\pi_{g}$-group $\Gamma$ so that $G=\Gamma / K$ as in the previous section) contains no parabolic transformations on the component $\Delta$. 
(iii) The group $G$ generated by $A(z)=z+2$ and $B(z)=\frac{z}{i z+1}$, is an example of a noded Schottky group of genus two which uniformizes a stable Riemann surface of genus two with three nodes, one of which is a dividing node.

Theorem 7 (Retrosection theorem for stable Riemann surfaces [9]) If $G$ is a noded Schottky group of genus $g$, then $\Omega^{e x t}(G) / G$ is a stable Riemann surface of genus $g$; the nodes of this surface being the projection of the parabolic fixed points. Reciprocally, every stable Riemann surface of genus $g$ can be uniformized by a noded Schottky group of genus $g$.

The above result asserts that the boundary points of $M_{g}$ inside the Deligne-Mumford's compactificaton $\widehat{M}_{g}$ can be also uniformized by noded Schottky groups, that is, only using some kind of stable Schottky groups.

Theorem 8 [[24]] Noded Schottky groups belong to the (algebraic) boundary of the corresponding Schottky space. They are exactly the geometrically finite boundary points.

The space of noded Schottky groups (containing the class of Schottky groups) $N S_{g}$ can be embedded into the space $\widehat{\mathbf{C}}^{3 g-3}$ using some correct choice of fixed points for a normalized collection of free generators of the groups [9]. The image of Schottky space $S_{g}$ is an open subset of $\widehat{\mathbf{C}}^{3 g-3}$. The natural holomorphic cover map (which is not regular) $\pi: S_{g} \rightarrow M_{g}$ extends continuously to a natural surjective map $\pi: N S_{g} \rightarrow \widehat{M}_{g}$. This construction is different to the one obtained in [8]; where they constructed an infinite coordinate system. As a matter of completeness, we recall the following commutator rigidity fact which is related to Torelli's theorem for closed Riemann surfaces.

Theorem 9 [[14]] Let $G$ be a noded Schottky group of genus $g \geq 2$. Then $G$ is uniquely determined by its commutator subgroup $[G, G]$.

\section{Extended Stable Schottky Groups}

In this section we describe uniformizations of stable Klein surfaces by means of extensions of stable Schottky groups (including noded Schottky groups) by anticonformal automorphisms of the regions of discontinuity on which they act. 


\subsection{Extended Stable Schottky groups}

Let us consider a stable Schottky uniformization $(Z, G, P: Z \rightarrow S)$ of some stable Riemann surface $S$ of genus $g$. If we have an anticonformal involution $\tau: S \rightarrow S$ which lifts to $Z$ under $P: Z \rightarrow S$ to an anticonformal homeomorphism $\widehat{\tau}: Z \rightarrow Z$, then the group $K=\langle G, \widehat{\tau}\rangle$ is called an extended stable Schottky group of genus $g$. The extended stable Schottky group $K$ uniformizes the stable Klein surface $S /\langle\tau\rangle$ in the sense that $(Z, K, Q: Z \rightarrow S /\langle\tau\rangle)$ is an uniformization, where $Q=h P$ and $h: S \rightarrow S /\langle\tau\rangle$ is the natural projection induced by the action of $\tau$ on $S$.

\subsection{Extended Noded Schottky groups}

An extended Kleinian group $G$ for which its index two orientation preserving half $G^{+}$is a noded Schottky group is called an extended noded Schottky group. As in the case of noded Schottky groups, we have that these groups can be considered as a subclass of extended stable Schottky groups which act on the Riemann sphere and not on a tree of Riemann spheres. We have that the stable Riemann surface $S=\Omega^{e x t}(G) / G^{+}$admits an anticonformal involution $\tau: S \rightarrow S$, which is induced by any element of $G-G^{+}$. The quotient $S /\langle\tau\rangle$ is a stable Klein surface which is uniformized by the extended Schottky group $G$, that is, $S /\langle\tau\rangle=\Omega^{\text {ext }}(G) / G$. In section 11.2 we observe that the stable Klein surface $S /\langle\tau\rangle$ is closed and without phantom nodes if and only if $G$ is freely acting on $\Omega^{e x t}(G)$; we say in this case that $G$ is a noded Klein-Schottky group.

As noded Schottky groups belong to the algebraic boundary of the corresponding Schottky space, we have, using similar arguments as in [24], that noded extended Schottky groups also belong to the algebraic boundary of the deformation spaces of a suitable extended Schottky group.

Proposition 1. Every noded extended Schottky group of genus $g$ belongs to the (algebraic) boundary of a suitable extended Schottky space.

Any two Schottky groups of the same genus are known to be quasiconformally equivalent, but we have seen in [15] that there are infinitely many topologically different noded Schottky groups of same genus. Moreover, only finitely many of these topological classes can be neoclassical and infinitely many are sufficiently complicated. In particular, if in example 2 below we use as Klein-Schottky group a cyclic group of order 2 generated by some imaginary reflection, we get the following. 
Theorem 10 There are infinitely many topologically different noded KleinSchottky groups of a fixed genus at least 2 . Infinitely many of these classes have a sufficiently complicated noded Schottky subgroup as index two orientation preserving half.

\section{Retrosection theorems for stable Klein surfaces}

We know that extended stable Schottky groups (in particular, extended noded Schottky groups) give uniformizations of stable Klein surfaces. In the following we proceed to observe that in fact every stable Klein surface can be so obtained.

\subsection{The non-hyperbolic cases: $g \in\{0,1\}$}

In example 1 we have listed the stable Klein surfaces of algebraic genus $g \in\{0,1\}$. We proceed to show a corresponding noded extended Schottky group that uniformizes each one of them.

(i) The closed disc is uniformized by the group generated by a reflection.

(ii) The real projective plane is uniformized by the group generated by an imaginary reflection.

(iii) The closed annuli is uniformized by the group generated by the reflections on two disjoint circles.

(iv) The real projective plane with one border is uniformized by the group generated by a reflection and an imaginary reflection, each one keeping invariant a circle, so that both circles are disjoint.

(v) The Klein bottle is uniformized by the group generated by two imaginary reflections, each one keeping invariant a circle, so that both circles are disjoint.

(vi) The closed disc with a phantom node is uniformized by the group generated by the translation $z \mapsto z+1$ and the reflection $z \mapsto \bar{z}$.

(v) The real projective plane with a phantom node is uniformized by the pseudo-parabolic $z \mapsto \bar{z}+1$.

(vi) The closed common region bounded by two tangent circles is uniformized by the reflections of two tangent circles. 


\subsection{The hyperbolic case}

We now consider the class of stable Klein surfaces of algebraic genus $g \geq 2$.

\subsubsection{Closed stable Klein surfaces without phantom nodes}

A closed stable Klein surface may only have nodes or phantom nodes. Let us observe that if $G$ is a noded Klein-Schottky group of genus $g$ (we denote by $G^{+}$its index two noded Schottky subgroup) and $\tau \in G$ is orientation reversing transformation, then we must have $\tau^{2} \in G^{+}$. Since $\tau$ is required not to have fixed points on $\Omega^{e x t}(G)$, we have that $\tau$ cannot be a pseudo-parabolic transformation. Similarly, if $\tau$ is a reflection, then we must have that its circle $C$ of fixed points of it must be contained in $\Lambda^{\text {ext }}(G) \subset \Lambda\left(G^{+}\right)$, in particular, $G^{+}$has $C$ as part of its limit set. In this situation, as $G^{+}$is a noded Schottky group, we must necessarily have that $G^{+}$contains parabolic transformations (otherwise it will be a Schottky group), whose fixed point will be necessarily fixed by $\tau$, a contradiction to the freely action property. It follows that the only possibilities for $\tau$ are to be either a imaginary reflection or a glide-reflection. In this way, if $G$ is a noded Klein-Schottky group of genus $g$, then it may only contains loxodromic transformations, parabolic transformations, imaginary reflections or glide-reflections. Let $S=\Omega^{e x t}(G) / G, S^{+}=\Omega^{e x t}(G) / G^{+}$and $\eta: S^{+} \rightarrow S^{+}$the anticonformal involution induced on $S^{+}$by any of the elements of $G-G^{+}$. The condition that on $\Omega^{\text {ext }}(G)$ none of the elements of $G-G^{+}$has fixed points gives the following facts:

(i) $\eta$ has no fixed points on $S^{+}-N\left(S^{+}\right)$; and

(ii) if $\eta$ has a node of $S^{+}$as fixed point, then we must have two parabolic transformations $A, B \in G^{+}$, with different fixed points, an element $C \in G^{+}$and an element $\tau \in G-G^{+}$so that $\tau$ and $C$ both send the fixed point of $A$ to the fixed point of $B$. In that case, we have that $\tau \circ C^{-1} \in G-G^{+}$will fix a parabolic fixed point belonging to $\Omega^{e x t}(G)$, a contradiction.

As a consequence of the above we have the following.

Theorem 11 If $G$ is a noded Klein-Schottky group of genus $g$, then $\Omega^{e x t}(G) / G$ is a closed stable Klein surface without phantom nodes.

In the following two examples we provide constructions of noded KleinSchottky groups which can be used together Klein-Maskit's combination 
theorems in order to produce a large family of noded Klein-Schottky groups.

Example 2. Let us start with a $(m, n)$-Klein-Schottky group $G_{1}$ and perform a free product from Klein-Maskit's combination theorems [23] with a noded Schottky group $G_{2}$ of genus $p$. The group $G=\left\langle G_{1}, G_{2}\right\rangle$, in this way constructed, is a noded Klein-Schottky group for which $S=$ $\Omega^{\text {ext }}(G) / G$ is the connected sum of the closed non-orientable Klein surface $S_{1}=\Omega\left(G_{1}\right) / G_{1}$ of topological genus $m+2 n$ and the stable Riemann surface $S_{2}=\Omega^{e x t}\left(G_{2}\right) / G_{2}$ of genus $p$.

Example 3. Let $\widehat{\tau}(z)=\bar{z}, \tau(z)=-\bar{z}, W(z)=i z, \Sigma$ be a circle, orthogonal to the unit circle, with center at a point $p>1$ and disjoint from $W(\Sigma)$, and $\tau_{1}$ the reflection on $\Sigma$. Set $\tau_{2}=W \circ \tau_{1} \circ W^{-1}, \tau_{3}(z)=-\tau_{1}(-z)$, $\tau_{4}(z)=-\tau_{2}(-z), r=\widehat{\tau} \circ \tau_{1}, A=\tau_{1} \circ \tau_{2}, B=\tau_{1} \circ \tau_{3}, C=\tau_{1} \circ \tau_{4}$ and $\eta=r \circ \tau$. Then we have that $r \circ A \circ r^{-1}=C^{-1}, r \circ B \circ r^{1}=B^{-1}$, $\tau \circ A \circ \tau=B^{-1} \circ A, \tau \circ B \circ \tau=B^{-1}, \tau \circ C \circ \tau=B^{-1} \circ C, \eta \circ A \circ \eta^{-1}=A$, $\eta \circ B \circ \eta^{-1}=B, \eta \circ C \circ \eta^{-1}=B \circ A^{-1}$ and $\eta^{2}=B$. The group

$$
G=\langle\eta, A\rangle=\langle\eta, A, B, C\rangle
$$

is a Klein-Schottky group uniformizing a closed non-orientable Klein surface isomorphic to $\#{ }^{4} \mathbf{R} \mathbf{P}_{2}$. Making $p$ to approach a suitable value $p_{\infty}>1$ (when the circles $\Sigma$ and $W(\Sigma)$ get tangent) the group $G$ converges algebraically to a noded Klein-Schottky group $G_{\infty}$ that uniformizes a stable surface $S_{\infty}$ with two non-dividing nodes, so that both of them divide $S_{\infty}$ into two components, each one a real projective plane.

Let us observe that using noded Klein-Schottky groups of types described in examples 2 and 3 and the first Klein-Maskit's combination theorem we may obtain noded Klein-Schottky groups uniformizing any topological type of a closed stable Klein surface without phantom nodes. In this way, quasiconformal deformation theorem permits to obtain the following.

Theorem 12 (Retrosection theorem for closed Klein surfaces without phantom nodes) If $S$ is a closed stable Klein surface with no phantom nodes, then there is a noded Klein-Schottky group so that $S=\Omega^{e x t}(G) / G$. 


\subsubsection{No phantom nodes nor half-nodes}

In the previous section we have considered the closed stable Klein surfaces without phantom nodes. In this section we proceed to consider those bordered compact stable Klein surfaces without phantom nodes and without half-nodes. Let us start with the following construction.

Example 4. Let $G$ be a noded Klein-Schottky group. By Theorem 11.2.1, the stable closed Klein surface $S=\Omega^{e x t}(G) / G$ has no phantom nodes. The nodal set of $S$ is denoted as before as $N(S)$. The components $S-N(S)$ are, each one, a Riemann surface with at most a finite number of punctures. Let us consider any component $R$ of $S-N(S)$. Inside $\Omega(G)$ there is a component $\Delta \subset \Omega$ over $R$. If $G_{\Delta}$ is the stabilizer of $\Delta$ in $G$, then $\Delta / G_{\Delta}=R$. Let $F \subset \Delta$ be a fundamental set for $G_{\Delta}$. We may choose a finite collection of pairwise disjoint circles $\Sigma_{1}, \ldots, \Sigma_{m}$, all of them inside $F$ and all of them bounding a common domain. For each circle $\Sigma_{j}$ we consider the reflection $\tau_{j}$ on it. The group $K=\left\langle G, \tau_{1}, \ldots, \tau_{m}\right\rangle$ is a noded extended Schottky group so that $\Omega^{e x t}(K) / K$ is topologically $S$ minus the interior of $m$ pairwise disjoint closed discs inside the component $R$.

The above construction, done with any collection of components of the complement of the nodal set, provides stable Klein surfaces having no phantom nodes nor half-nodes and permits to obtain the following.

Theorem 13 (Retrosection theorem for compact Klein surfaces without phantom nodes nor half-nodes).

If $S$ is a compact stable Klein surface without phantom nodes and without half-nodes, then there is a noded extended Schottky group so that $S=\Omega^{e x t}(G) / G$. 


\subsubsection{Adding some phantom nodes}

In the previous sections we have considered compact and closed stable Klein surfaces without phantom nodes. We proceed to find uniformizations under the presence of them. We first start with the following construction.

Example 5. Let $F$ be a Fuchsian group, acting on a disc $\Delta$, so that $\Delta / F$ is the Riemann sphere with $n \geq 3$ punctures. Set $G=\langle F, \tau\rangle$, where $\tau$ is the reflection on the boundary circle of $\Delta$. As $F$ is a noded Schottky group, the group $G$ is a noded extended Schottky group that uniformizes the stable Klein surface given by the Riemann sphere with exactly $n$ phantom nodes.

If we use, in the first Klein-Maskit's combination theorem, noded extended Schottky groups as in the previous sections and groups as in the above example, then we may produce at least 3 phantom nodes on each component of the nodal set of a compact stable surface. This permits us to obtain the following.

Theorem 14 (Retrosection theorem for compact Klein surfaces without half-nodes) If $S$ is a compact (including the closed ones) stable Klein surface with no half-nodes and so that on each component of the complement of the nodes there are none or at least 3 phantom nodes, then there is a noded extended Schottky group so that $S=\Omega^{e x t}(G) / G$.

\subsubsection{The general situation}

Let us assume we have a pair $(S, \tau)$, where $S$ is some stable Riemann surface of genus $g \geq 2$, and $\tau: S \rightarrow S$ is an anticonformal involution. In order to obtain a uniformization of the stable Klein surface $S /\langle\tau\rangle$ by some noded extended Schottky group or some noded stable Schottky group, we need to answer the following problem: Is there either a noded Schottky group or a stable Schottky group uniformizing $S$ for which $H=\langle\tau\rangle$ lifts?, equivalently, Is $H$ of Schottky type? Clearly, if there is a positive answer to this question, then the group generated by the noded Schottky group (or the stable Schottky group) and the lifted anticonformal automorphism will be an extended noded Schottky group (or an extended stable Schottky group) uniformizing $S /\langle\tau\rangle$. 
By a fattening process of the nodes of $S$ we obtain a closed orientable surface $\Sigma$, a deformation $\sigma: \Sigma \rightarrow S$ and an orientation reversing homeomorphism $j: \Sigma \rightarrow \Sigma$ for which (i) $\sigma \circ j=\tau \circ \sigma$ and (ii) each node $p \in N(S)$ is the image of a simple closed curve $\alpha_{p} \in \Sigma$. We may also assume that if $\tau(p)=p$, then either :

(1) $j$ acts as the identity on $\alpha_{p}$;

(2) $j$ has exactly two fixed points on $\alpha_{p}$.

We may give a Riemann surface structure to $\Sigma$ so that $j$ is an anticonformal involution. Also, we may assume that each loop $\alpha_{p}$ is a simple closed geodesic. As we have already consider the case when $S /\langle\tau\rangle$ is closed without phantom nodes, we may assume from now on that $\operatorname{Fix}(\tau) \neq \emptyset$. The following was already proved in [12].

Theorem 15 Let $S$ be a stable Riemann surface of genus $g$ and $\tau$ : $S \rightarrow S$ an anticonformal involution so that $\operatorname{Fix}(\tau) \neq \emptyset$. Then there is a stable Schottky uniformization $(Z, H, P: Z \rightarrow S)$ of $S$ for which $\tau$ lifts, that is, there is an anticonformal automorphism $\tau^{*}: Z \rightarrow Z$ such that $P \circ \tau^{*}=\tau \circ P$.

Corollary 2 (Retrosection theorem for stable Klein surfaces). Every stable Klein surface can be uniformized by a suitable extended stable Schottky group.

Question 1. Is the above result true by only using extended noded Schottky groups?

\subsection{Proof of theorem 11.2.4}

Let us consider a stable Riemann surface $S$ (with nodes) of genus $g \geq 2$ with an anticonformal involution given by $\tau: S \rightarrow S$ acting with fixed points. Denote by

$N=\left\{P_{1}, \ldots, P_{r}, P_{r+1}=\tau\left(P_{1}\right), \ldots, P_{2 r}=\tau\left(P_{r}\right), Q_{1}=\tau\left(Q_{1}\right), \ldots, Q_{l}=\tau\left(Q_{l}\right)\right\}$

the set of nodes of $S$, where $r+l>0$, and $Q_{1}, \ldots, Q_{l}$ are exactly the fixed nodes by $\tau$.

If we consider a neighborhood $W$ of a node $Q_{j}$ so that $W-\left\{Q_{j}\right\}$ consists of two pairwise disjoint punctured discs, then there are two possibilities: (i) 
$\tau$ permutes these two discs, or (ii) $\tau$ keeps invariant each of the to punctures discs. In situation (ii), on each of the two punctured discs there are two pairwise disjoint arcs, each one connecting the puncture to the exterior border, so that each of these consists of only fixed points of $\tau$.

By fattening the nodes (see, for instance, [21]) as previously stated, we obtain a closed orientable surface $\Sigma$ of genus $g$ with an orientation reversing homeomorphism $j: \Sigma \rightarrow \Sigma$ of order two satisfying conditions (1) and (2) above. It is well known that we can give to $\Sigma$ a Riemann surface structure so that $j$ is an anticonformal involution. The hipothesis of the theorem asserts that $j$ has fixed points.

Set $S_{0}=\Sigma, S_{1}=S, \tau_{0}=j$ and $\tau_{1}=\tau$. The results from either [21] or theorem 9.1 assert that we can find a regular B-group $G$ (a noded $\pi_{g}$ group), with simply connected invariant component $\Delta_{0}$ so that, for $k=0,1$, $S_{k}=\Delta_{k} / G$, where $\Delta_{1}=\Omega^{e x t}(G)-\Delta_{0}$. B. Maskit [28] has shown that $G$ must have parabolic transformations; otherwise, it will be a quasifuchsian group and, consequently, $S$ will have no nodes. Let us denote by $P(G)$ the collection of parabolic fixed points of the noded $\pi_{g}$ group $G$. Each parabolic transformation in $G$ is necesarilly an accidental one (see [23] for the definition of accidental parabolic transformation) as $S_{0}$ is a closed Riemann surface.

For each $k \in\{0,1\}$, we set $\pi_{k}: \Delta_{k} \rightarrow S_{k}=\Delta_{k} / G$ the natural quotient map induced by the action of $G$. We recall that a set $X$ is precisely invariant under a subgroup $\Gamma$ of a Kleinian group $G$ if $\gamma(X)=X$ for all $\gamma \in \Gamma$ and $\gamma(X) \cap X=\emptyset$ for all $\gamma \in G-\Gamma$. We need the following lemma.

Lemma. There are continuous surjective maps $\tilde{\sigma}: \Delta_{0} \rightarrow \Delta_{1}$ and $\sigma: S_{0} \rightarrow$ $S_{1}$, such that

(1) $\pi_{1} \tilde{\sigma}=\sigma \pi_{0}$;

(2) $\tilde{\sigma}$ commutes with each element of $G$;

(3) the preimage under $\tilde{\sigma}$ of each parabolic fixed point $p$ is a simple arc precisely invariant under the stabilizer of the point $p$ in $G$;

(4) all these arcs are pairwise disjoint;

(5) the map $\tilde{\sigma}$ is a homeomorphism from the complement of the above arcs onto $\Delta_{1}-P(G)=\Omega(G)-\Delta_{0}$; and

(6) the map $\sigma$ is a deformation, where the preimage of each node is exactly the simple loop obtained by projecting the respective ( $G$ class) arc in (3) via $\pi_{0}$. 
Proof. For each node $p$ of $S_{1}$ we choose a maximal parabolic cyclic subgroup $H_{p}$ of $G$, with fixed point $x_{p} \in P(G)$, so that $\pi_{1}\left(x_{p}\right)=p$. We can choose a simple arc $\alpha_{p}$ in $\Delta_{0}$ which is precisely invariant under $H_{p}$ in $G$. The end points of such an arc are the same and equal to $x_{p}$. This arc will project onto a simple loop on $S_{0}$. Now consider the $G$-translates of that arc. Continuing this construction for each node of $S_{1}$ we obtain a collection $F$ of pairwise disjoint arcs, projecting onto a disjoint family of simple loops on $S_{0}$. Let $X$ be a connected component of $\Delta_{1}-P(G)$. This component has stabilizer $G_{X}$ in $G$. We have that $X / G_{X}$ is one of the connected components of $S_{1}-N\left(S_{1}\right)$. There is a unique component of $\Delta_{0}-F$, say $Y$, with stabilizer $G_{X}$. Moreover, $Y$ is precisely invariant under $G_{X}$. Let us consider the connected component $Y / G_{X}$ of $S_{0}-\pi_{0}(F)$. Since both components $X$ and $Y$ are simply connected, we have that $X / G_{X}$ and $Y / G_{X}$ are homeomorphic surfaces with boundary. We can choose an orientation preserving homeomorphism $\sigma: Y / G_{X} \rightarrow X / G_{X}$ and an orientation preserving homeomorphism $\tilde{\sigma}: Y \rightarrow X$, such that $\tilde{\sigma}$ commutes with $G_{X}$ (by the uniqueness of universal covering space) and such that $\pi_{1} \tilde{\sigma}=\sigma \pi_{0}$. Now we can proceed to glue all this mappings to obtain a homeomorphism $\tilde{\sigma}: \Delta_{0}-F \rightarrow \Delta_{1}-P(G)$ and a homeomorphism $\sigma: S_{0}-\pi_{0}(F) \rightarrow S_{1}-\pi_{1}(P(G))$ satisfying (1) and (2). If we extend the map $\tilde{\sigma}$ to each arc $c \in F$ by sending it to its end point, then we have the desired map. The map $\sigma$ can be extended to the above loops continuously preserving the condition (1).

Now we continue with the proof of Theorem 11.2.4. The simply connectivity of $\Delta_{0}$ ensures the existence of an anticonformal automorphism $\eta_{0}: \Delta_{0} \rightarrow \Delta_{0}$ such that $\pi_{0} \eta_{0}=\tau_{0} \pi_{0}$. Since $\operatorname{Fix}\left(\tau_{0}\right) \neq \emptyset$, we may assume that $\eta_{0}$ has fixed points on $\Delta_{0}$, in particular, $\eta_{0}^{2}=I$ (as consequence of Riemann mapping theorem).

The set of fixed points of $\eta_{0}$ is a simple geodesic arc (respect to the natural hyperbolic structure on $\Omega(G)$, $A x\left(\eta_{0}\right)$, with end points in the boundary of $\Delta_{0}$, and $\eta_{0}$ permutes the two components of $\Delta_{0}-A x\left(\eta_{0}\right)$.

Consider a connected component $X_{1}$ of $S_{1}-N\left(S_{1}\right)$. Set $X_{2}=\tau_{1}\left(X_{1}\right)$. Fix some lifting $\tilde{X}_{1}$ of $X_{1}$ in $\Omega(G)-\Delta_{0}$ and let $G_{1}$ the stabilizer of $\tilde{X}_{1}$ in $G$. Now consider the connected component $\tilde{Y}_{1}$ of $\Delta_{0}-F$ for which $G_{1}$ is its stabilizer (we have that $\tilde{\sigma}\left(\tilde{Y}_{1}\right)=\tilde{X}_{1}$ ). Set $\tilde{Y}_{2}=\eta_{0}\left(\tilde{Y}_{1}\right), G_{2}$ its stabilizer in $G$, and let us consider the connected component $\tilde{X}_{2}$ of $\Omega(G)-\Delta_{0}$ with stabilizer $G_{2}$ (which is equal to $\tilde{\sigma}\left(\tilde{Y}_{2}\right)$ ). The equality $\pi_{1} \tilde{\sigma}=\sigma \pi_{0}$ asserts $\pi_{1}\left(\tilde{X}_{2}\right)=X_{2}$. Both, $\tilde{X}_{1}$ and $\tilde{X}_{2}$ are simply connected and, in particular, we may lift $\tau_{1}$ to an anticonformal map $\eta_{1}: \tilde{X}_{1} \rightarrow \tilde{X}_{2}$ so that $\eta_{1} \tilde{\sigma}=\tilde{\sigma} \eta_{0}$. 
In particular, $\eta_{1}$ induces the same isomorphism $\theta: G_{1} \rightarrow G_{2}$ induced by $\eta_{0}$. Proceeding in this way, we may find an anticonformal automorphism $\eta_{1}: \Delta_{1}-P(G) \rightarrow \Delta_{1}-P(G)$ such that $\tilde{\sigma} \eta_{0}=\eta_{1} \tilde{\sigma}$. Since the map $\eta_{1}$ permutes the maximal parabolic cyclic subgroups of $G$, we have that this map extends continuously to all of $P(G)$. By gluing both maps $\eta_{0}$ and $\eta_{1}$, we get a new map $\eta: \Omega(G)^{e x t} \rightarrow \Omega(G)^{e x t}$ satisfying the following:

(i) $\eta$ is an anticonformal automorphism of $\Omega^{e x t}(G)$;

(ii) it induces an algebraic automorphism of $G$; and

(iii) $\eta^{2} \in G$.

Let (i) $K$ be the group generated by $G$ and $\eta$, (ii) $\tilde{T}$ be the torsion part of $K$, and (iii) $T$ the index two subgroup of conformal automorphisms of $\tilde{T}$. We have that $T$ is a subgroup of $G$.

Since $\Delta_{0}$ is simply connected, we have from Heltai's result in [7] that $\Delta_{0} / T$ is a planar surface and $G / T$ is a Schottky group of genus $g$ with region of discontinuity $\Delta_{0} / T$. It follows that $G / T$ (acting on $Z=\Delta_{1} / T$ ) is a stable Schottky group of rank $g$, defining a stable Schottky uniformization

of $S_{1}=S$ for which the anticonformal involution $\tau$ lifts. This ends the proof of Theorem 11.2.4.

\section{References}

[1] L. V. Ahlfors. Finitely generated Kleinian groups, Amer. J. Math. 86 (1964), 413-423; 87 (1965), 759.

[2] N. L. Alling and N. Greenleaf, N. Foundations of the theory of Klein surfaces. Lect. Notes in Math. 219, Springer-Verlag, (1971).

[3] L. Bers. Automorphic forms for Schottky groups,. Adv. in Math. 16, pp. 332-361, (1975).

[4] J. Button. All Fuchsian Schottky groups are classical Schottky groups. Geometry \& Topology Monographs 1: The Epstein birthday schrift, pp. 117-125, (1998).

[5] V. Chuckrow. On Schottky groups with applications to Kleinian groups. Annals of Math. 88, pp. 47-61, (1968). 
[6] D. Hejhal. On Schottky and Teichmüller spaces. Advances in Math. 15, pp. 133-156, (1975).

[7] B. Heltai. Symmetric Riemann surfaces, torsion subgroups and Schottky coverings. Proc. of the Amer. Math. Soc. 100, pp. 675-682, (1987).

[8] L. Gerritzen and F. Herrlich. The extended Schottky space. J. Reine Angew. Math. 389, pp. 190-208, (1988).

[9] R. A. Hidalgo The Noded Schottky Space. Proc. London Math. Soc. 3, pp. 385-403, (1996).

[10] R. A. Hidalgo. Noded Fuchsian groups I. Complex variables, 36, pp. 45-66, (1998).

[11] R. A. Hidalgo. Kleinian groups with an invariant Jordan curve: Jgroups. Pacific Journal of Math. 169, pp. 291-309, (1995).

[12] R. A. Hidalgo. Schottky uniformization of stable symmetric Riemann surfaces. Notas de la Sociedad Matemática de Chile (NS) No. 1, pp. 82-91, (2001).

[13] R. A. Hidalgo. Automorphisms of Schottky type. Ann. Acad. Scie. Fenn. Mathematica 30, pp. 183-204, (2005).

[14] R. A. Hidalgo. Noded function groups. Complex geometry of groups (Olmué, 1998), 209-222, Contemp. Math., 240, Amer. Math. Soc., Providence, RI, (1999).

[15] R. A. Hidalgo and B. Maskit. On Neoclassical Schottky groups. Trans. of the Amer. Math. Soc. 358, pp. 4765 - 4792, (2006).

[16] R. A. Hidalgo and B. Maskit. On Klein-Schottky groups. Pacific J. of Math. (2) 220, pp. 313-328, (2005).

[17] R. A. Hidalgo and B. Maskit. Extended Schottky groups. Preprint.

[18] P. Koebe. Über die Uniformisierung der Algebraischen Kurven II. Math. Ann. 69 (1910),1-81, (1910).

[19] P. Koebe. Über die Uniformisierung reeller algebraischer Kurven. Nachr. Akad. Wiss. Goettingen, pp. 177-190, (1907).

[20] I.Kra. Automorphic forms and Kleinian groups, Benjamin, New York, (1972). 
[21] I. Kra. Horocyclic coordinates for Riemann surfaces and moduli spaces of Kleinian groups. J. Amer. Math. Soc. 3 (1990), 499-578.

[22] A. Marden. Schottky groups and circles. Contribution to Analysis, a collection of papers dedicated to Lipman Bers (L.V. Ahlfors a.o., Eds.), Academic Press, New York 1974, 273-278.

[23] B. Maskit. Kleinian Groups. Springer-Verlag, 1988.

[24] B. Maskit. On free Kleinian groups. Duke Math. J. 48 (1981), 755-765.

[25] B. Maskit. A characterization of Schottky groups. J. d'Analyse Math. 19 (1967), 227-230.

[26] B. Maskit. On the classification of Kleinian groups:I. Koebe groups. Acta Math. 135, pp. 249-270, (1975).

[27] B. Maskit. On the classification of Kleinian groups:II. Signatures. Acta Math. 138, pp. 17-42, (1977).

[28] B. Maskit. On a class of Kleinian groups. Ann. Acad. Sci. Fenn. Ser. A I Math. (1969).

[29] B. Maskit. Self-maps on Kleinian groups. Amer. J. Math. XCIII, pp. 840-856, (1971).

[30] D. Mumford. Stability of projective varieties, L'enseignement math. 23, pp. 39-110, (1977).

[31] S. Nag, The Complex Analytic Theory of Teichmüller Spaces (Wiley, New York, (1988)).

[32] H. Sato. On a paper of Zaroow. Duke Math. J. 57, pp. 205-209, (1988).

[33] H. Yamamoto. An example of a nonclassical Schottky group. Duke Math. J. 63, pp. 193-197, (1991).

[34] R. Zarrow. Classical and nonclassical Schottky groups. Duke Math. J. 42, pp. 717-724, (1975). 
Rubén Hidalgo

Departamento de Matemática,

Universidad Técnica Federico Santa María,

Valparaíso,

Chile

e-mail : ruben.hidalgo@usm.cl 